\title{
International Group for Reducing Inappropriate Medication Use \& Polypharmacy (IGRIMUP): Position Statement and 10 Recommendations for Action
}

\author{
Dee Mangin ${ }^{1,9}$ (D) Gülistan Bahat ${ }^{2} \cdot$ Beatrice A. Golomb $^{3} \cdot$ Laurie Herzig Mallery $^{4}$ • \\ Paige Moorhouse $^{4} \cdot$ Graziano Onder $^{5} \cdot$ Mirko Petrovic $^{6} \cdot$ Doron Garfinkel $^{7,8}$
}

Published online: 14 July 2018

(C) The Author(s) 2018

\begin{abstract}
Globally, the number of drug prescriptions is increasing causing more adverse drug events, which is now a significant cause of mortality, morbidity, and disability that has reached epidemic proportions. The risk of adverse drug events is correlated to very old age, multiple comorbidities, dementia, frailty, and limited life expectancy, with the major contributor being polypharmacy. Each characteristic alters the risk-benefit balance of medications, typically reducing anticipated benefits and amplifying risk. Current clinical guidelines are based on evidence proven in younger/healthier adult populations using a single disease model and their application to older adults with multimorbidity, in whom testing has not been conducted,
\end{abstract}

Dee Mangin

mangind@mcmaster.ca

Gülistan Bahat

gbahatozturk@yahoo.com

Beatrice A. Golomb

bgolomb@ucsd.edu

Laurie Herzig Mallery

laurie.mallery@nshealth.ca

Paige Moorhouse

Paige.Moorhouse@nshealth.ca

Graziano Onder

graziano.onder@rm.unicatt.it

Mirko Petrovic

mirko.petrovic@ugent.be

Doron Garfinkel

dgarfink@netvision.net.il

1 Department of Family Medicine, Faculty of Health Sciences, McMaster University, 100 Main Street West, Hamilton, ON, Canada yields a different risk-benefit prospect and makes inappropriate medication use and polypharmacy inevitable. Applying inappropriate clinical practice guidelines to older adults is antithetical to good healthcare, is likely to increase health inequity, and is associated with substantial negative clinical, economic, and social implications for health systems. The casualties are on the scale of a war or epidemic, yet are usually invisible in measures of healthcare quality and formal recommendations. Radical and rapid action is required to achieve a better quality of life for older populations and to remain true to the principles of medical professionalism and evidence-based medicine that place patients' interests and autonomy at the fore. This first International Group for Reducing Inappropriate Medication Use \& Polypharmacy position statement briefly details the

2 Division of Geriatrics, Department of Internal Medicine, Istanbul Medical School, Istanbul University, Istanbul, Turkey

3 Department of Medicine, University of California San Diego, San Diego, CA, USA

4 Division of Geriatric Medicine, Department of Medicine, Dalhousie University, Halifax, NS, Canada

5 Department of Geriatrics, Università Cattolica del Sacro Cuore, IRCCS Fondazione Policlinico Universitario A. Gemelli, Rome, Italy

6 Department of Internal Medicine, Section of Geriatrics, Faculty of Medicine and Health Sciences, Ghent University, Ghent, Belgium

7 Wolfson Medical Center, Holon, Israel

8 Homecare Hospice Israel Cancer Association, Holon, Israel

9 Department of General Practice, University of Otago, Christchurch, New Zealand 
causes, consequences, and extent of inappropriate medication use and polypharmacy. This article outlines current strategies to reduce inappropriate medication use, provides evidence for their effect, and then proposes recommendations for moving forward with 10 recommendations for action and 12 recommendations for research. We conclude that an urgent integrated effort to reduce inappropriate medication use and polypharmacy should be a leading global target of the highest priority. The cornerstone of this position statement from the International Group for Reducing Inappropriate Medication Use \& Polypharmacy is the understanding that without evidence of definite relevant benefit, when it comes to prescribing, for many older patients 'less is more'. This approach differs from most other current recommendations and guidance in medical care, as the focus is on what, when, and how to stop, rather than on when to start medications/interventions. Disrupting the framework that indiscriminately applies standard guidelines to older adults requires a new approach that better serves patients with multimorbidity. This transition requires a shift in medical education, research, and diagnostic frameworks, and re-examination of the measures used as quality indicators. In achieving this objective, we promote a return to some of the original concepts of evidence-based medicine: which considers scientific data (where it exists), clinical judgment, patient/family preference, and context. A shift is needed: from the current model that focuses on single conditions to one that simultaneously considers multiple conditions and patient priorities. This approach reframes the clinician's role as a professional providing care, rather than a disease technician.

\section{Key Points}

Polypharmacy is an urgent issue that requires a coordinated global effort to provide medical care systems that better serve patients with multimorbidity.

This transition requires a shift in medical education, research, and diagnostic frameworks, and reexamination of the measures used as quality indicators.

This position statement from the International Group for Reducing Inappropriate Medication Use \& Polypharmacy briefly summarizes the current situation and provides a call to action for moving forward, proposing 10 recommendations for action and 12 recommendations for research.

\section{Background and Objectives}

Over recent decades, the number of drug prescriptions has increased globally. As a result of their increased number of chronic diseases and geriatric syndromes, older adults are the main drug users, consuming over one-third of medications used in the USA $[1,2]$. Compared with a younger population, older adults are at higher risk of medicationrelated adverse events [3]. Vulnerability characteristics such as very old age, multiple co-morbidities, dementia, frailty, and limited life expectancy markedly alter the riskbenefit balance of medications. These characteristics are often accompanied by impaired medication clearance and reduced physiological reserve [4] and, in combination with polypharmacy (traditionally understood as taking five or more long-term medications), significantly reduce benefit and amplify the risk of most drug therapies, thus making adverse drug events (ADEs) and inappropriate medication use almost inevitable. There is a robust literature and reviews that already describe the negative medical, economic, and social consequences of inappropriate medication use and polypharmacy (IMUP), which are summarized in Table 1.

Many risk factors, such as elevated cholesterol, glucose, and blood pressure, lose much of their negative portent in older age [37-39] and some risk factors reverse to become predictors of better outcomes. Therefore, treating markerdefined conditions to target goals developed for younger people may be ineffective or, worse, damaging [26, 40-42].

While noting the importance and risks of under-prescribing in some settings, the focus of this position paper is over-prescribing. Medical errors, including ADEs are now leading causes of death, with many events yet to be recognized [24, 43, 44]. These casualties are on the scale of a war or epidemic; yet, this iatrogenic 'epidemic' is nearly invisible in guideline recommendations and quality measures. Just as with a war or epidemic, radical and rapid action is required to place the interests and autonomy of older populations at the fore, to achieve the best quality of life possible. This radical action is not against drugs or their prescription, nor against drug companies, but against the many forces that, in aggregate, can lead to damaging or unwanted overmedication of the elderly.

As the portion of older subpopulations with complex morbidity expands, many warn that we are ill equipped intellectually, economically, and professionally to face this global challenge, in general, and the IMUP epidemic, in particular [36, 45-50]. We therefore believe that current global trends in healthcare require assertive and coordinated action against IMUP. We present the first position statement of IGRIMUP (International Group for Reducing 
Table 1 Negative outcomes and hazards of inappropriate medication use and polypharmacy

\begin{tabular}{ll}
\hline 1. & Drug-specific adverse effects \\
2. & Drug-drug interaction \\
3. & Drug-disease interaction \\
4. & Cognitive impairment/delirium [5-8] \\
5. & Weight loss, malnutrition [9, 10] \\
6. & Falls [11-15] \\
7. & Hip fractures [12] \\
8. & Urinary incontinence [16] \\
9. & Functional impairment, immobility [17, 18] \\
10. & Hospitalization [17, 19-26] \\
11. & Nursing home, long-term care placement [20-24, 27] \\
12. & Decrease in quality of life [27] \\
13. & Death [27] \\
14. & Reduced treatment adherence [28] \\
15. & Increased cost to the health system and to the individual [17, 20-24, 29-36]
\end{tabular}

Inappropriate Medication Use \& Polypharmacy) on the international co-operative effort, and recommendations for actions needed to prevent and counter IMUP and its drivers globally.

\section{Main Approaches for Reducing Inappropriate Medication Use and Polypharmacy and Their Clinical Efficacies}

Many studies report on tools and strategies that have attempted to reduce IMUP. However, the global increase in the IMUP epidemic indicates that their efficacy is limited. There is inadequate information about the beneficial effect of these approaches on health outcomes, such as mortality, morbidity, function, cognition, patient well-being, healthcare services' utilization, and cost. A 2010 cohort study of multiple medication discontinuation using a 'consecutive patient' sampling frame [50] is often referenced in the literature on polypharmacy as supporting the benefical health outcomes of using a general approach to drug discontinuation in community-dwelling patients. A further cohort study suggests long-term health benefits from drug discontinuation, compared with controls who declined deprescribing [51]. These prospective studies achieved medication reductions and apparent benefits to health outcomes while measuring potential adverse effects, but were not randomized controlled trials. Studies in long-term residential care settings show that medications can be reduced but have yet to demonstrate beneficial outcomes, aside from a reduction in falls in those who have already had a fall $[52,53]$. Other evidence of potential benefit is based on retrospective assessments of explicit lists of 'drugs to avoid', comparing these with admissions for ADEs to determine the sensitivity in predicting such events. One review concluded that evidence for health outcomes from pharmacist and physician based interventions to reduce polypharmacy is limited and conflicting, and recommended randomized controlled trials evaluating multidisciplinary interventions and clinical outcomes across different settings [54].

We present the main methods suggested to improve IMUP and briefly note where successful clinical outcomes are achieved by some of them. These methods are usually categorized as explicit criteria-based tools (some computer assisted) and implicit judgment-based tools.

\subsection{Computer-Assisted Digital Tools}

A variety of computer programs have been developed in many countries to ease application of the explicit criteria to detect IMUP. In countries with full medication information in an electronic medical record, these systems may provide alarms that alert clinicians to a variety of potential drug interactions as they turn on their computers and try to prescribe new drugs [55]. However, a systematic review of 10 studies of computerized physician order entry with clinical decision support showed a mixed effect on the reduction of ADEs [56]. There are suggestions that automatic messages, particularly where there are many alerts and more repeated alerts, lead to reminder fatigue and insensitivity [57]. Others warn that relying too much on computers, where decision support is single-disease oriented, may be misleading or harmful to older patients $[50,58]$. 


\subsection{Explicit Tools}

Explicit tools include lists of drugs to avoid or specific indicators of inappropriate medication use. A comparison of seven tools failed to show close similarities [59]. The most widely used are the Beers criteria and the Screening Tool of Older Persons' potentially inappropriate Prescriptions/Screening Tool to Alert to Right Treatment (STOPP/START) [60-66]. Both were updated in 2015. The criteria divide potentially inappropriate medications into: medications to avoid prescribing; to avoid in certain circumstances; and to be used with caution. Following the Beers initiative in 1991, other country-specific lists were established: in Germany, PRISCUS (Latin for 'old and venerable') [67] and the Fit for the Aged (FORTA); in France, a consensus panel list [68]; in Norway, the Norwegian General Practice (NORGEP) list [69]; in Canada, the Improving Prescribing in the Elderly Tool (IPET) [70]; and others [71, 72], including in Australia [73, 74] and Thailand [75]. In the USA, Assessing Care of Vulnerable Elders (ACOVE) [76, 77], the Health Care Financing Administration expert consensus panel drug utilization review criteria [78], and Geriatric Risk Assessment MedGuide (GRAM) [79] have also been developed.

Other scales specifically identify the anticholinergic burden of medications. Among the multiple scales developed to assess anticholinergic burden, the Drug Burden Index seemed to best predict adverse health outcomes [80].

The Beers criteria has not yet been evaluated in a randomized controlled trial and, therefore, the extent of the lists effect in decreasing ADEs, morbidity, mortality, hospitalization and/or costs is uncertain. Analysis in a prospective cohort study shows modest sensitivity in predicting hospital admissions as a result of ADEs [65]. Similarly, while applying STOPP/START criteria in older inpatients significantly improves the appropriateness of prescribing [64] and could potentially reduce avoidable ADEs that may cause urgent hospitalization [65, 81], the tool has not yet been prospectively tested in the primary care setting and its effect on long-term outcomes is yet to be described. These lists are limited by their single drug/ disease-oriented approach, and require regular updating.

Applying the Geriatric Risk Assessment MedGuide (GRAM) tool in long-term care was proven efficacious in reducing the rate of delirium, hospitalizations, and mortality resulting from ADEs in newly admitted residents [79]. However, other clinical outcomes were not assessed and it has not yet been prospectively tested in the primary care setting.

'Fit for the Aged Criteria' (FORTA) combines both negative and positive labeling based on individual indications. It ranks drugs into four groups depending on evidence for safety, efficacy, and overall age appropriateness: (A) indispensable with obvious benefit; (B) proven efficacy but limited effects or possible safety concerns; (C) questionable efficacy or safety; (D) avoid [82]. A randomized trial to validate FORTA in hospitalized geriatric patients was associated with an improvement in medication quality and a reduction in ADEs [83]. Its effect on overall patient outcomes is not yet tested beyond feasibility studies [84].

Other evidence of the potential benefit of explicit tools is based on retrospective assessments that use lists of 'drugs to avoid' and compare these with admissions for ADEs to determine the sensitivity in predicting (and therefore their potential to prevent) such events. These studies provide retrospective epidemiological data, but again, do not supply adequate unbiased proof that is required to understand the benefits of prospective application of these lists. There are other ongoing positive efforts to develop frameworks that (1) guide drug review; (2) reduce the harms of polypharmacy; and (3) advance single drug class deprescribing guidelines [50, 63, 85-87].

\subsection{Implicit Approaches}

To judge medication appropriateness, implicit approaches take into consideration research data, clinical circumstances, and patient/family preferences [88, 89]. Implicit approaches are less algorithmic and require much more time, knowledge, and judgment. This more complex approach is better suited to multimorbidity and to a shared decision-making model. However, the complexity of this approach creates problems for study design and relatively few studies have been carried out to assess efficacy and safety.

\subsubsection{Comprehensive Geriatric Assessment}

Comprehensive Geriatric Assessment (CGA) is a thorough evaluation of the older patient's characteristics, cognition, function, medical conditions, and social situation, which is used to identify problems and appropriately manage them through individualized care plans [90]. Though there is variation in the execution of the CGA, drug review is an integral component, with the minimal requirement of medication count and assessment, preferably at each visit and at least annually [76]. If time limitation is a concern, the minimal requirement is to focus on those drugs with the highest risk or highest benefit [28]. Multidisciplinary team approaches using the CGA in frail hospitalized older adults have been shown to significantly reduce serious ADEs and IMUP, when compared with usual care [91]. Therefore, the CGA in itself may serve as an important tool for reducing IMUP $[88,89]$. 


\subsubsection{Medication Appropriateness Index}

The Medication Appropriateness Index is a judgmentbased process measure of prescribing appropriateness that assesses 10 elements of prescribing: indication, effectiveness, dose, correct directions, practical directions, drugdrug interactions, drug-disease interactions, duplication, duration, and cost. The Medication Appropriateness Index is the only implicit tool with validated inter-rater reliability [92]. In heterogeneous smaller studies, the Index reduced ADEs compared with usual care [93], but the tool has not been extensively used in larger settings.

\subsubsection{Prescribing Optimization Method}

The Prescribing Optimization Method is based on six questions: Is undertreatment present and the addition of medication indicated? Does the patient adhere to his/her medication schedule? Which drugs can be withdrawn or which drugs are inappropriate for the patient? Which adverse effects are present? Which clinically relevant interactions are to be expected? Should the dose, dose frequency, or form of drug be adjusted? [94].

\subsubsection{SMART (Specific, Measurable, Acceptable, Realistic, and Time-framed) Tool}

The SMART (Specific, Measurable, Acceptable, Realistic and Time-framed) Tool is a method for reviewing complex geriatric drug regimens. It consists of 10 questions that draw attention to the appropriateness and safety of the drug plan. Therapeutic objectives are developed to improve quality of life, which the authors of the tool believe lead to a better understanding of geriatric clinical pharmacology, the special needs of older patients, and appropriate use of healthcare resources [95].

\subsubsection{Patient-Focused Drug Surveillance}

Patient-focused drug surveillance was developed in Swedish nursing homes [96]. The intervention involved a physician-led, patient-focused approach that optimizes medication therapy and reduces polypharmacy by taking the patient's health conditions into account. This approach advocates for a discussion of the benefits and risks of drug therapy with frail older people, accompanied by close monitoring and re-evaluation.

\subsubsection{CRIME (CRIteria to Assess Appropriate Medication Use Among Elderly Complex Patients)}

CRIME (CRIteria to assess appropriate Medication use among Elderly complex patients) offers 19 explicit recommendations on pharmacological treatments of only 5 common conditions (diabetes mellitus, hypertension, congestive heart failure, atrial fibrillation, and coronary heart disease). In line with vulnerability characteristics, CRIME takes into consideration complex aspects of aging (i.e. limited life expectancy, functional/cognitive impairments, and geriatric syndromes) that may negatively affect a drug's benefit/risk ratio and therefore reduce its efficacy [97].

\subsubsection{PATH (Palliative and Therapeutic Harmonization) Program}

The PATH (Palliative and Therapeutic Harmonization) program was developed in Canada [98]. One goal of this program is to achieve frailty-specific treatment guidelines to replace conventional clinical practice guidelines using an evidence review of common chronic conditions, such as hypertension, diabetes, and statin use for the prevention of cardiovascular disease [99-101]. The guidelines consider the clinical relevance of commonly accepted outcomes when there is frailty and suggest that even the outcome of reduced mortality may not be relevant with frailty owing to multiple competing risks for mortality. Based on an evidence review, the panel of experts conclude that rigid blood pressure, serum glucose, and cholesterol targets may be harmful in patients with severe frailty.

\subsubsection{0-Step Discontinuation Guide}

The 10-Step Discontinuation Guide was formulated by Scott et al. and synthesizes suggestions and guidance raised in other frameworks [28, 86, 102, 103]. The guide recommends a case-specific framework for each patient, with confirmed face validity showing that doctors who used the guide suggested more drugs for discontinuation for a hypothetical case [104].

\subsubsection{Good Palliative Geriatric Practice Algorithm}

The Good Palliative Geriatric Practice Algorithm is a proactive process that simultaneously discontinues as many 'non-life saving' medications as possible where there is an absence of evidence in older people. Attention is paid to a patient's circumstances and preference for care, and to providing follow-up monitoring (see Fig. 1) [50]. In nursing homes, the discontinuation of 2.8 drugs per patient led to a significant $24 \%$ reduction in mortality and $18 \%$ in referral to acute care facilities [105]. In communitydwelling elderly individuals, discontinuation of 4.4 drugs per patient led to an improvement of global health and well-being in $88 \%$ of the patients; only $2 \%$ of the discontinued drugs had to be re-administered and no significant 
Fig. 1 Good Palliative Geriatric Practice Algorithm
Discuss the following with the patient/guardian

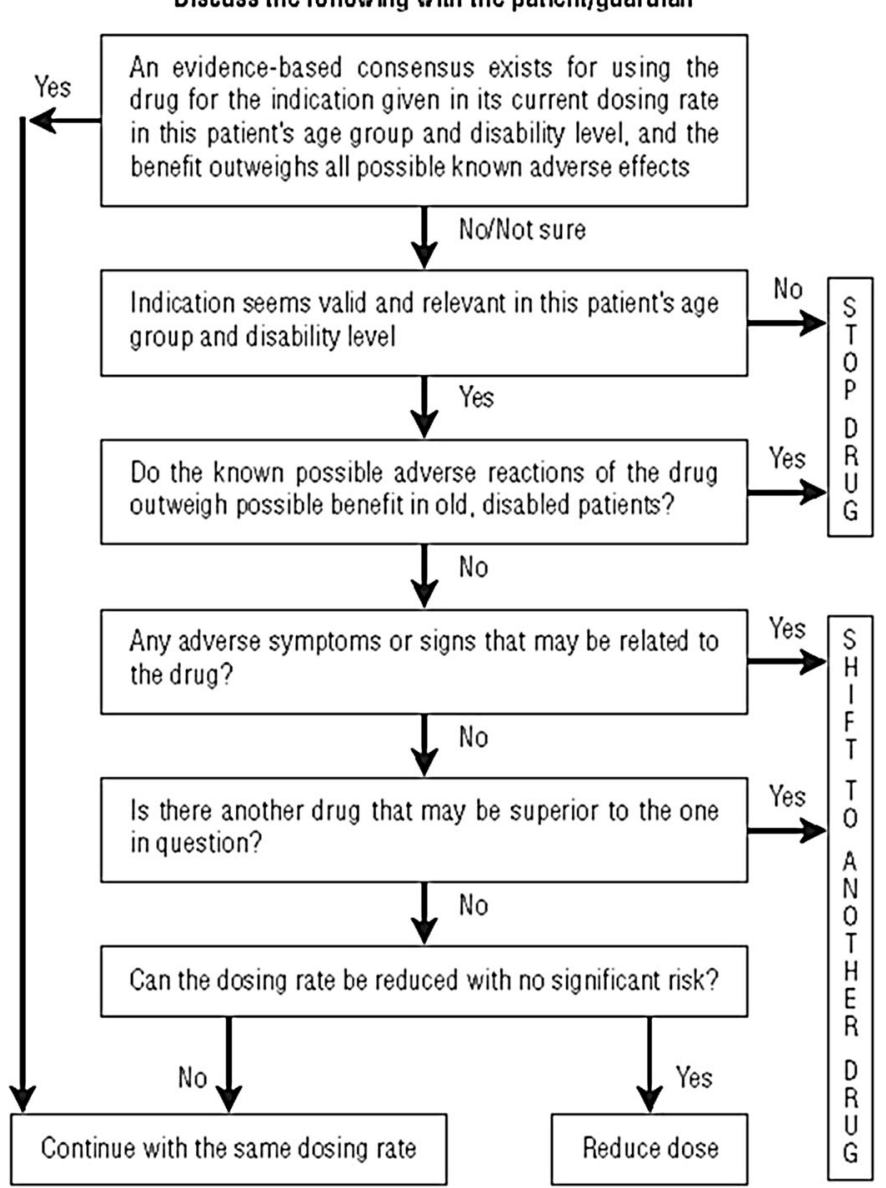

adverse events were recorded as a result of deprescribing [50]. This approach is translatable into any setting; but requires knowledge of the limitations of standard guidelines. A longer follow-up ( $>3$ years) cohort study showed significantly better clinical outcomes in older people undergoing multiple deprescribing, compared with those who did not, suggesting benefits are sustained [51].

\section{Moving Forward}

"Where is the Wisdom We Have Lost in Knowledge?"

This phrase, from TS Elliott's Choruses from The Rock, reminds us that the competent use of multiple medicines requires the application of wisdom, based on a critical understanding of the limitations and generalizability of research and of patient circumstances and preferences. The original definition of evidence-based medicine (EBM) stated: "Evidence-based medicine is the integration of best research evidence with clinical expertise and patient values" [106]. The EBM diagram shows how these concepts are integrated using clinical judgment. More recently evidence-based medicine has been reconfigured as single disease guideline adherence, whereby evidence largely encourages clinicians to start medications, but the integration of clinical judgment and patient-centered care are lost. In this situation variation may be seen as poor practice.

The cornerstone of this position statement is that without evidence of definite and relevant benefit, when prescribing for many older patients 'less is more'. The approach differs from standard guidance as the focus here is on what, when, and how to stop, rather than on when to start medications/ interventions. The scope can be extended beyond frailty to include many subpopulations with multimorbidity, disability, or limited life expectancy. It extends beyond the list-based approach of any explicit tool.

Our principal goal is to promote a return to the original concept of EBM and restore the physician's role to one of a professional providing care, rather than a disease or algorithm technician. This role requires integration of patient preference and context, clinical judgment, and scientific data (where it exists), using a drug prescribing approach that can be customized to each older adult in a fashion aligned with patient-centered medicine ideals. A return to 
the more nuanced EBM framework, as described, will produce variation in care; but consistency in care is not synonymous with best care.

Most elderly patients have not had a formal review of their long-term medications and there is evidence that most patients, if asked, would like to reduce their medications and there is some evidence that patient-directed approaches to reducing single medicines can be effective [107-109]. The potential benefits of medication review to healthcare systems and patients are substantial and include reduced drug costs, ADEs, morbidity, and hospitalizations. Reducing IMUP should be a shared goal of the highest urgency that requires an integrated international effort.

There is a growing call for a medical system that better serves patients with multimorbidity $[36,58,110,111]$. Barriers to addressing inappropriate medication use derive from the limitations of research; an inadequate framework for the clinical care of patients with polypharmacy, performance measures and incentives that focus on starting but not stopping medications; commercial influences; health system design; inadequate education about medication risks; and insufficient funding, all of which impact prescribers, pharmacists, and patients. Addressing these barriers will require changes to medical education, research practice, diagnostic frameworks, measures of quality, and policy [36, 58, 112-114].

\subsection{Recommendations of the International Group for Reducing Inappropriate Medication Use \& Polypharmacy}

We make 10 recommendations for action and 12 recommendations for research.

Individual and System Approaches to Inappropriate Medication Use and Polypharmacy

1. Review the medications of all older adults with an eye to deprescribing, particularly those who are vulnerable to the adverse effects of medication.

Patients with polypharmacy deserve special attention. Consideration of medication reduction does not mean that medication reduction is appropriate for everyone. Likewise, after stopping a medication, the need to reintroduce that medication should not be seen as a failure.

2. Before initiating a potentially 'appropriate' medication, consider the validity of the evidence based on patient characteristics and preferences.

Clinicians should bear in mind that disease-specific guidelines (and perceptions of 'under-prescribing') are driven by data from trials that almost always exclude (or do not separately evaluate) older adults, particularly those with vulnerability characteristics. When faced with polypharmacy, patients can be overwhelmed with the burden of treatment, which can compromise adherence. Thus, medications may need to be prioritized, recognizing that using all potentially 'helpful' medications may not be appropriate. Deprescribing based on the paradigm described may lead to labels of 'under-prescribing', but needs to be reframed as ideal prescribing in this context.

3. Consider each medication for potential withdrawal, extending beyond standardized lists.

Many unnecessary and inappropriate medications for an individual are not included in lists of 'potentially inappropriate medications' [50].

4. Employ mixed implicit and explicit approaches to polypharmacy.

Explicit criteria contribute to the detection of IMUP and are useful support systems, but inadequate on a stand-alone basis. Drugs-to-avoid criteria are insufficiently accurate, focus only on the more common causes of ADEs, and may provide false reassurance: prescribing 10-15 'non-list' medications to patients is still likely to do more harm than good [50, 59, 115-117].

5. Address the underrepresentation of older patients in clinical trials.

Older adults are largely excluded from randomized controlled trials; and those that are included are non-representative of an older population with multimorbidity [118-122]. Randomized controlled trial findings may, thus, overestimate the benefit-risk balance in favor of increased prescribing in the group least able to tolerate it. This approach is antithetical to good healthcare and is likely to increase health inequity [111, 123-125]. Beyond recruitment strategies, the impact of underrepresentation/misrepresentation of older patients in trials might be at least partially addressed by incorporating stratified randomization and analysis, where possible. However, trial participation is disproportionately burdensome to patients with physical and cognitive limitations, who may also be unable to consent. Thus, approaches to enhance the enrollment of older adults must be complemented by explicit recognition that trial findings often overstate benefit over risk in older adults.

We also need a better understanding of the dose-effect curve and benefit/risk ratio of drugs used by older adults and especially for the subpopulation of older adults with frailty and multimorbidity. While observational studies contribute to our understanding of how treatment effects may be different in older age groups, on balance multiple concurrent medication use in older adults is a global experiment with little data collection. We need data on the overall health effects of this type of prescribing. 
6. Acknowledge and address commercial influences on polypharmacy: trial results should not be implemented in older adults unless access to all available patientlevel data is provided. Appropriate outcome measures should be required before licensing indications that include older populations.

The degree to which commercial interests can potentially distort scientific data is well documented [126-131]. Trials can be structured to provide commercially favorable results and there is limited access to patient-level trial and adverse-event data, which are grounds for precautionary prescribing [132]. Use of intermediate outcomes, publication bias, and overhyping of new or immature research results by media and pharmaceutical companies result in a research narrative that overestimates efficacy, underestimates harms, and fuels IMUP [133-135]. Evidence bias is commonly compounded by biased interpretation, where key opinion leaders have industry conflicts of interest [136].

7. Medical education needs a stronger focus on IMUP and its potential negative impact. Education about generalist approaches to multimorbidity should teach prioritization skills and aim to improve the clinician's understanding of the strengths and weaknesses of evidence and how best to apply standard models of care to vulnerable older adults with multimorbidity.

Currently these topics are inadequately emphasized in the curriculum to doctors, nurses, and pharmacists [137] with insufficient education about the harm of polypharmacy, specific drug-class ADEs, the importance of medication reviews, and how specialty prescribing may increase IMUP and lead to prescribing cascades [138, 139].

8. Medical training should review methods to stop treatments and provide equal attention to drug side effects and benefits.

When systematically discontinuing medications, physicians face multiple barriers, including fear of lawsuits, uncertainty about the best approach to combat IMUP, and lack of evidence-based studies on why, when, and how to stop medications [58, 140, 141]. Most patients would be agreeable to reducing medication numbers, if recommended. However, when elderly patients/families want to reduce the drug load, they are pressured not to discontinue medications [141, 142]. Providing more balanced communication on risk and benefit, such as reviewing numbers needed to treat along with the number not benefiting, may help. Likewise, the generalizability of accepted outcomes such as all-cause mortality for elderly individuals could be discussed.

9. When patients have multimorbidity, the single disease model (and its incentivization) should be spurned.

About half of people over 65 years of age have at least three coexisting chronic conditions and one in five has five or more [143, 144]. The single disease approach with adherence to clinical guidelines for each illness make polypharmacy and inappropriate medication use inevitable. Boyd et al. [123] demonstrates this clearly: a patient experiencing five chronic conditions will receive 19 doses of 12 different medications, taken at five times during the day, carrying the risk of ten attendant interactions and adverse events. Quality measures that assess care on a single disease basis might rate this compliance with guidelines as "good care" but what appears measurably better is meaningfully worse for the patient. When performance pay or professional re-certifications are linked to guideline adherence, physicians may feel coerced into persuading patients to follow treatments that do not serve their best interests.

A single disease-by-disease approach obscures the multimorbid patient's individual pattern of symptoms and overrides their preferences for care. Data also suggest that treatments that focus on preventing single diseases at the end of the survival curve may simply change the cause of death and morbidity without making life longer or better; in essence, altering the manner of dying rather than the quality of living [145]. Efforts to reframe guidance in terms of multimorbidity also note that support for preventive drugs for single diseases weakens as life expectancy decreases and that the additional benefit provided by individual drugs may reduce (or reverse) when combined [146].

10. Decisions in older complex patients should routinely consider expected survival and quality of life, giving the highest priority to patient/family preferences.

One physician should co-ordinate decisions within a shared framework (preferably in a generalist setting suited to consideration of multimorbidity and polypharmacy ideally with a pharmacist partner). Care for patients with multimorbidity should balance the burden of treatment, potential to benefit, potential harms, and personal priorities. The term 'personalized medicine' could be re-harnessed to describe these considerations.

Many important research and clinical needs arise from these 10 recommendations; we have proposed 12 polypharmacy research priorities in Table 2 . 
Table 212 polypharmacy research priorities

1. Clarify the risks and benefits of different approaches to reduce polypharmacy using patient-relevant outcomes such as all-cause mortality, morbidity, function cognitive status, and healthcare utilization and cost

2. Identify subgroups that will most benefit and those that may not

3. Determine the reversibility of the negative health effects of inappropriate medication use and polypharmacy

4. Clarify the effect of stopping treatments that have been used for many years

5. Consider the effects of different clinical care funding models on strategies to address polypharmacy

6. Define core important outcomes in treatment trials for drugs used in multimorbidity that assess risks and benefits based on patient and system-relevant domains

7. Research, synthesize, and review the relative benefits and risks of different treatment options for individual chronic diseases, including nonpharmaceutical treatments

8. Research, synthesize, and review the evidence on how and in whom to stop individual drugs

9. Develop tools to assess the capacity for, and burden of, treatment in multimorbidity

10. Develop tools that can aid the detection and management of drug adverse effects

11. Assess different approaches to incorporating patient priorities, including in cognitive impairment

12. Research optimal dosing in older adults

\section{Conclusions}

Reducing IMUP should be a high priority target. The morbidity and mortality attributed to the adverse effects of IMUP underscore this imperative. Studies suggest benefit from judicious deprescribing that may be equivalent to benefits achieved from medical treatments. Our goal is to promote a return to the original concept of EBM that integrates patient preference, context, clinical judgment, and scientific data (where it exists). There is a pressing need to reconceptualize the framework of medical care to better serve patients with multimorbidity, which will require shifts in medical education, quality measures, and policy. To reverse this epidemic of iatrogenic morbidity and mortality, an integrated global effort from health professionals, policy makers, and consumers is required.

Acknowledgements The authors acknowledge Hannah Lamb, McMaster University, for her invaluable and elegant work in helping edit the manuscript, and the reviewers whose thoughtful comments improved the final version of the manuscript. Note on the International Group for Reducing Inappropriate Medication Use \& Polypharmacy (IGRIMUP): all authors, along with many others worldwide, work with the shared goal of reducing the negative health and economic effects of inappropriate medication use. The idea of creating an international group to support this work via information sharing, research, and communication was born in a symposium at the 2009 International Association of Gerontology and Geriatrics Congress in Paris. The association was established at the 2013 International Association of Gerontology and Geriatrics Congress in Seoul. While the authors represent the IGRIMUP early members, IGRIMUP now includes more than 100 members from 26 countries and the efforts of this wider group are acknowledged.

\section{Compliance with Ethical Standards}

Funding No funding was received for the preparation of this article.
Conflict of Interest All authors have completed the Author Declaration Form and have no conflicts directly relevant to the content of this article.

Open Access This article is distributed under the terms of the Creative Commons Attribution-NonCommercial 4.0 International License (http://creativecommons.org/licenses/by-nc/4.0/), which permits any noncommercial use, distribution, and reproduction in any medium, provided you give appropriate credit to the original author(s) and the source, provide a link to the Creative Commons license, and indicate if changes were made.

\section{References}

1. Kaufman DW, et al. Recent patterns of medication use in the ambulatory adult population of the United States: the Slone survey. JAMA. 2002;287(3):337-44.

2. Lombardi TP, Kennicutt JD. Promotion of a safe medication environment: focus on the elderly and residents of long-term care facilities. Medsc Pharm. 2001;2(1):23-8.

3. Thomsen LA, et al. Systematic review of the incidence and characteristics of preventable adverse drug events in ambulatory care. Ann Pharmacother. 2007;41(9):1411-26.

4. Topinkova E. Aging, disability and frailty. Ann Nutr Metab. 2008;52(Suppl. 1):6-11.

5. Gill TM, Robison JT, Tinetti ME. Predictors of recovery in activities of daily living among disabled older persons living in the community. J Gen Intern Med. 1997;12(12):757-62.

6. Alagiakrishnan K, Wiens C. An approach to drug induced delirium in the elderly. Postgrad Med J. 2004;80(945):388-93.

7. Larson EB, et al. Adverse drug reactions associated with global cognitive impairment in elderly persons. Ann Intern Med. 1987;107(2):169-73.

8. Moore AR, O'Keeffe ST. Drug-induced cognitive impairment in the elderly. Drugs Aging. 1999;15(1):15-28.

9. Huffman GB. Evaluating and treating unintentional weight loss in the elderly. Am Fam Physician. 2002;65(4):640-50.

10. Jyrkka $\mathrm{J}$, et al. Association of polypharmacy with nutritional status, functional ability and cognitive capacity over a three-year period in an elderly population. Pharmacoepidemiol Drug Saf. 2011;20(5):514-22. 
11. Gnjidic D, et al. Polypharmacy cutoff and outcomes: five or more medicines were used to identify community-dwelling older men at risk of different adverse outcomes. J Clin Epidemiol. 2012;65(9):989-95.

12. Lai SW, et al. Polypharmacy correlates with increased risk for hip fracture in the elderly: a population-based study. Medicine (Baltimore). 2010;89(5):295-9.

13. Leipzig RM, Cumming RG, Tinetti ME. Drugs and falls in older people: a systematic review and meta-analysis: II. Cardiac and analgesic drugs. J Am Geriatr Soc. 1999;47(1):40-50.

14. Leipzig RM, Cumming RG, Tinetti ME. Drugs and falls in older people: a systematic review and meta-analysis: I. Psychotropic drugs. J Am Geriatr Soc. 1999;47(1):30-9.

15. Thapa PB, et al. Antidepressants and the risk of falls among nursing home residents. N Engl J Med. 1998;339(13):875-82.

16. Talasz H, Lechleitner M. Polypharmacy and incontinence. Z Gerontol Geriatr. 2012;45(6):464-7.

17. Akazawa M, et al. Potentially inappropriate medication use in elderly Japanese patients. Am J Geriatr Pharmacother. 2010;8(2):146-60.

18. Lopez-Sendon JL, Mena MA, de Yebenes JG. Drug-induced parkinsonism in the elderly: incidence, management and prevention. Drugs Aging. 2012;29(2):105-18.

19. Onder G, et al. Adverse drug reactions as cause of hospital admissions: results from the Italian Group of Pharmacoepidemiology in the Elderly (GIFA). J Am Geriatr Soc. 2002;50(12):1962-8.

20. Ernst FR, Grizzle AJ. Drug-related morbidity and mortality: updating the cost-of-illness model. J Am Pharm Assoc (Wash). 2001;41(2):192-9.

21. Bates DW, et al. The costs of adverse drug events in hospitalized patients: Adverse Drug Events Prevention Study Group. JAMA. 1997;277(4):307-11.

22. Fick DM, et al. Updating the Beers criteria for potentially inappropriate medication use in older adults: results of a US consensus panel of experts. Arch Intern Med. 2003;163(22):2716-24.

23. Johnson JA, Bootman JL. Drug-related morbidity and mortality: a cost-of-illness model. Arch Intern Med. 1995;155(18):1949-56.

24. Perry DP. When medicine hurts instead of helps. Consult Pharm. 1999;14:1326-30.

25. Gurwitz JH, et al. Incidence and preventability of adverse drug events in nursing homes. Am J Med. 2000;109(2):87-94.

26. Han $\mathrm{BH}$, et al. Effect of statin treatment vs usual care on primary cardiovascular prevention among older adults: the ALLHATLLT randomized clinical trial. JAMA Intern Med. 2017;177(7):955-65.

27. Jyrkka J, et al. Patterns of drug use and factors associated with polypharmacy and excessive polypharmacy in elderly persons: results of the Kuopio 75+ study: a cross-sectional analysis. Drugs Aging. 2009;26(6):493-503.

28. Steinman MA, Hanlon JT. Managing medications in clinically complex elders: "There's got to be a happy medium". JAMA. 2010;304(14):1592-601.

29. Fu AZ, et al. Potentially inappropriate medication use and healthcare expenditures in the US community-dwelling elderly. Med Care. 2007;45(5):472-6.

30. Hug BL, et al. The costs of adverse drug events in community hospitals. Jt Comm J Qual Patient Saf. 2012;38(3):120-6.

31. Rochon PA, TJ, Gill SS, Gurwitz JH. In: Halter JB, Tinetti ME, Studenski S, High KP, Asthana S, editors. Hazzard's Geriatric Medicine and Gerontology. New York (NY): McGraw-Hill; 2009. p. 289-302.
32. Bootman JL, Harrison DL, Cox E. The health care cost of drugrelated morbidity and mortality in nursing facilities. Arch Intern Med. 1997;157(18):2089-96.

33. Hoonhout LH, et al. Direct medical costs of adverse events in Dutch hospitals. BMC Health Serv Res. 2009;9:27.

34. Rottenkolber D, Hasford J, Stausberg J. Costs of adverse drug events in German hospitals: a microcosting study. Value Health. 2012;15(6):868-75.

35. Santibanez-Beltran $S$, et al. Economic cost of polypharmacy in the elderly in primary health care. Rev Med Inst Mex Seguro Soc. 2013;51(2):192-9 (in Spanish).

36. Mangin D, Heath I, Jamoulle M. Beyond diagnosis: rising to the multimorbidity challenge. BMJ. 2012;44:e3526.

37. de Ruijter W, et al. Use of Framingham risk score and new biomarkers to predict cardiovascular mortality in older people: population based observational cohort study. BMJ. 2009;338:a3083.

38. Golomb BA, Bui AK. Fasting glucose positively predicts word memory performance in older men. Circulation. 2014;130(Suppl. 2):A13365.

39. BA, G. The starving cell: metabolic syndrome as an adaptive process. Available from: Nature Precedings; 2011.

40. The Action to Control Cardiovascular Risk in Diabetes. Study Group. Effects of intensive glucose lowering in type 2 diabetes. N Engl J Med. 2008;358(24):2545-59.

41. Tinetti ME, et al. Antihypertensive medications and serious fall injuries in a nationally representative sample of older adults. JAMA Intern Med. 2014;174(4):588-95.

42. Lipska KJ, et al. National trends in US hospital admissions for hyperglycemia and hypoglycemia among Medicare beneficiaries, 1999 to 2011. JAMA Intern Med. 2014;174(7):1116-24.

43. Lazarou J, Pomeranz BH, Corey PN. Incidence of adverse drug reactions in hospitalized patients: a meta-analysis of prospective studies. JAMA. 1998;279(15):1200-5.

44. Makary MA, Daniel M. Medical error: the third leading cause of death in the US. BMJ. 2016;353:i2139.

45. Garfinkel D. Geriatric boom catastrophe: a major medical, economic and social nightmare of the 21 st century. Proceedings of the 16th Congress of the International Association of Gerontology 1997; 364.

46. Boyle P. Global burden of cancer. Lancet. 1997;349(Suppl. 2):23-6.

47. Ershler WB. Geriatrics and medical oncology: finding the common ground. J Gerontol A Biol Sci Med Sci. 1997;52(6):M327-8.

48. Garfinkel D. The tsunami in 21 st century healthcare: the agerelated vicious circle of co-morbidity. J Nutr Health Aging. 2013;17(Suppl. 1):SS24, 227-C1, S96-7.

49. Bahat $\mathrm{G}$, et al. Comorbidities, polypharmacy, functionality and nutritional status in Turkish community-dwelling female elderly. Aging Clin Exp Res. 2014;26(3):255-9.

50. Garfinkel D, Mangin D. Feasibility study of a systematic approach for discontinuation of multiple medications in older adults: addressing polypharmacy. Arch Intern Med. 2010;170(18):1648-54.

51. Garfinkel D. Poly-de-prescribing to treat polypharmacy: efficacy and safety. Ther Adv Drug Saf. 2018;9(1):25-43.

52. Potter K, et al. Deprescribing in frail older people: a randomised controlled trial. PLoS One. 2016;11(3):e0149984.

53. Page AT, et al. Deprescribing in older people. Maturitas. 2016;91:115-34.

54. Gnjidic D, et al. Deprescribing trials: methods to reduce polypharmacy and the impact on prescribing and clinical outcomes. Clin Geriatr Med. 2012;28(2):237-53. 
55. Lynch T. Management of drug-drug interactions: considerations for special populations: focus on opioid use in the elderly and long term care. Am J Manag Care. 2011;17(Suppl. 11):S293-8.

56 . Wolfstadt JI, et al. The effect of computerized physician order entry with clinical decision support on the rates of adverse drug events: a systematic review. J Gen Intern Med. 2008;23(4):451-8.

57. Ancker JS, et al. Effects of workload, work complexity, and repeated alerts on alert fatigue in a clinical decision support system. BMC Med Inform Decis Mak. 2017;17(1):36.

58. Garfinkel D, Ilhan B, Bahat G. Routine deprescribing of chronic medications to combat polypharmacy. Ther Adv Drug Saf. 2015;6(6):212-33.

59. Chang CB, Chan DC. Comparison of published explicit criteria for potentially inappropriate medications in older adults. Drugs Aging. 2010;27(12):947-57.

60. Beers $\mathrm{MH}$, et al. Explicit criteria for determining inappropriate medication use in nursing home residents: UCLA Division of Geriatric Medicine. Arch Intern Med. 1991;151(9):1825-32.

61. American Geriatrics Society. 2015 updated Beers Criteria for potentially inappropriate medication use in older adults. J Am Geriatr Soc. 2015;63(11):2227-46.

62. Gallagher P, Barry P, O'Mahony D. Inappropriate prescribing in the elderly. J Clin Pharm Ther. 2007;32(2):113-21.

63. Gallagher P, O’Mahony D. STOPP (Screening Tool of Older Persons' potentially inappropriate Prescriptions): application to acutely ill elderly patients and comparison with Beers' criteria. Age Ageing. 2008;37(6):673-9.

64. Gallagher PF, O'Connor MN, O'Mahony D. Prevention of potentially inappropriate prescribing for elderly patients: a randomized controlled trial using STOPP/START criteria. Clin Pharmacol Ther. 2011;89(6):845-54.

65. Hamilton $\mathrm{H}$, et al. Potentially inappropriate medications defined by STOPP criteria and the risk of adverse drug events in older hospitalized patients. Arch Intern Med. 2011;171(11):1013-9.

66. O'Mahony D, et al. STOPP/START criteria for potentially inappropriate prescribing in older people: version 2. Age Ageing. $2015 ; 44(2): 213-8$.

67. Holt S, Schmiedl S, Thürmann PA. Potentially inappropriate medications in the elderly: the PRISCUS list. Dtsch Ärztebl Int. 2010;107(31-32):543-51.

68. Laroche ML, Charmes JP, Merle L. Potentially inappropriate medications in the elderly: a French consensus panel list. Eur J Clin Pharmacol. 2007;63(8):725-31.

69. Rognstad S, et al. The Norwegian General Practice (NORGEP) criteria for assessing potentially inappropriate prescriptions to elderly patients: a modified Delphi study. Scand J Prim Health Care. 2009;27(3):153-9.

70. Naugler CT, et al. Development and validation of an improving prescribing in the elderly tool. Can J Clin Pharmacol. 2000;7(2):103-7.

71. McLeod P, et al. Defining inappropriate practices in prescribing for elderly people: a national consensus panel. CMAJ. 1997;156:385-91.

72. Rancourt C, et al. Potentially inappropriate prescriptions for older patients in long-term care. BMC Geriatr. 2004;4:9.

73. Basger BJ, Chen TF, Moles RJ. Inappropriate medication use and prescribing indicators in elderly Australians: development of a prescribing indicators tool. Drugs Aging. 2008;25(9):777-93.

74. Basger BJ, Chen TF, Moles RJ. Validation of prescribing appropriateness criteria for older Australians using the RAND/ UCLA appropriateness method. BMJ Open. 2012;2(5):e001431.

75. Winit-Watjana W, Sakulrat P, Kespichayawattana J. Criteria for high-risk medication use in Thai older patients. Arch Gerontol Geriatr. 2008;47(1):35-51.
76. Shrank WH, Polinski JM, Avorn J. Quality indicators for medication use in vulnerable elders. $\mathrm{J}$ Am Geriatr Soc. 2007;55(Suppl. 2):S373-82.

77. Shekelle P, Maclean C, Morton S, Wenger N. ACOVE quality indicators. Ann Intern Med. 2001;135:653-67.

78. Hanlon JT, et al. Use of inappropriate prescription drugs by older people. J Am Geriatr Soc. 2002;50(1):26-34.

79. Lapane KL, et al. Effect of a pharmacist-led multicomponent intervention focusing on the medication monitoring phase to prevent potential adverse drug events in nursing homes. J Am Geriatr Soc. 2011;59(7):1238-45.

80. Salahudeen MS, Hilmer SN, Nishtala PS. Comparison of anticholinergic risk scales and associations with adverse health outcomes in older people. J Am Geriatr Soc. 2015;63(1):85-90.

81. Tosato M, et al. Potentially inappropriate drug use among hospitalised older adults: results from the CRIME study. Age Ageing. 2014;43(6):767-73.

82. Wehling M. Multimorbidity and polypharmacy: how to reduce the harmful drug load and yet add needed drugs in the elderly? Proposal of a new drug classification: fit for the aged. J Am Geriatr Soc. 2009;57(3):560-1.

83. Wehling M, et al. VALFORTA: a randomised trial to validate the FORTA (Fit fOR The Aged) classification. Age Ageing. 2016;45(2):262-7.

84. Kuhn-Thiel AM, Weiss C, Wehling M. Consensus validation of the FORTA (Fit fOR The Aged) list: a clinical tool for increasing the appropriateness of pharmacotherapy in the elderly. Drugs Aging. 2014;31(2):131-40.

85. BPAC. A practical guide to stopping medicines in older people. Best Pract J. 2010;27;10-23.

86. Scott IA, et al. Minimizing inappropriate medications in older populations: a 10-step conceptual framework. Am J Med. 2012;125(6):529-37.e4.

87. Farrell B, et al. What are priorities for deprescribing for elderly patients? Capturing the voice of practitioners: a modified delphi process. PLoS One. 2015;10(4):e0122246.

88. Onder G, et al. Strategies to reduce the risk of iatrogenic illness in complex older adults. Age Ageing. 2013;42(3):284-91.

89. Petrovic M, van der Cammen T, Onder G. Adverse drug reactions in older people: detection and prevention. Drugs Aging. 2012;29(6):453-62.

90. Ellis G, et al. Comprehensive geriatric assessment for older adults admitted to hospital: meta-analysis of randomised controlled trials. BMJ. 2011;343:d6553.

91. Schmader KE, et al. Effects of geriatric evaluation and management on adverse drug reactions and suboptimal prescribing in the frail elderly. Am J Med. 2004;116(6):394-401.

92. Hanlon JT, et al. A method for assessing drug therapy appropriateness. J Clin Epidemiol. 1992;45(10):1045-51.

93. Schmader KE, et al. Inappropriate prescribing and health outcomes in elderly veteran outpatients. Ann Pharmacother. 1997;31(5):529-33.

94. Drenth-van Maanen AC, et al. Prescribing optimization method for improving prescribing in elderly patients receiving polypharmacy: results of application to case histories by general practitioners. Drugs Aging. 2009;26(8):687-701.

95. Vogt-Ferrier N. Reviewing a complicated geriatric drug regimen. Eur Geriatr Med. 2010;1(3):198-202.

96. Olsson IN, Curman B, Engfeldt P. Patient focused drug surveillance of elderly patients in nursing homes. Pharmacoepidemiol Drug Saf. 2010;19(2):150-7.

97. Onder G, et al. Recommendations to prescribe in complex older adults: results of the CRIteria to assess appropriate Medication use among Elderly complex patients (CRIME) project. Drugs Aging. 2014;31(1):33-45. 
98. Moorhouse P, Mallery LH. Palliative and therapeutic harmonization: a model for appropriate decision-making in frail older adults. J Am Geriatr Soc. 2012;60(12):2326-32.

99. Dalhousie University. Faculty of Medicine continuing medical education. 8 Jan 2014. Available from: http://cme.medicine.dal. ca/ADS.htm. Accessed 2 May 2018.

100. Mallery LH, et al. Evidence-informed guidelines for treating frail older adults with type 2 diabetes: from the Diabetes Care Program of Nova Scotia (DCPNS) and the Palliative and Therapeutic Harmonization (PATH) program. J Am Med Dir Assoc. 2013;14(11):801-8.

101. Mallery LH, et al. Promoting higher blood pressure targets for frail older adults: a consensus guideline from Canada. Cleve Clin J Med. 2014;81(7):427-37.

102. Scott IA, et al. Deciding when to stop: towards evidence-based deprescribing of drugs in older populations. Evid Based Med. 2013;18(4):121-4.

103. Pollock M, Bazaldua OV, Dobbie AE. Appropriate prescribing of medications: an eight-step approach. Am Fam Physician. 2007;75(2):231-6.

104. Scott IA, Gray LC, Martin JH, et al. Effects of a drug minimisation guide on prescribing intentions in elderly persons with polypharmacy. Drugs Aging. 2012;29:659-67.

105. Garfinkel D, Zur-Gil S, Ben-Israel J. The war against polypharmacy: a new cost-effective geriatric-palliative approach for improving drug therapy in disabled elderly people. Israel Med Assoc J. 2007;9(6):430-4.

106. Sackett DL, et al. Evidence based medicine: what it is and what it isn't. BMJ. 1996;312(7023):71-2.

107. Canadian Institute for Health Information. Seniors and the health care system: what Is the impact of multiple chronic conditions? 2011. Available from: https://secure.cihi.ca/free_ products/air-chronic_disease_aib_en.pdf. Accessed 2 May 2018.

108. Reeve E, et al. People's attitudes, beliefs, and experiences regarding polypharmacy and willingness to deprescribe. J Am Geriatr Soc. 2013;61(9):1508-14.

109. Tannenbaum $\mathrm{C}$, et al. Reduction of inappropriate benzodiazepine prescriptions among older adults through direct patient education: the EMPOWER cluster randomized trial. JAMA Intern Med. 2014;174(6):890-8.

110. Starfield B. Threads and yarns: weaving the tapestry of comorbidity. Ann Fam Med. 2006;4(2):101-3.

111. Tinetti ME, Fried T. The end of the disease era. Am J Med. 2004;116(3):179-85.

112. May C, Montori VM, Mair FS. We need minimally disruptive medicine. BMJ. 2009;339:b2803.

113. Mangin D, Heath I. Multimorbidity and quaternary prevention (P4). Revista Brasileira de Medicina de Família e Comunidade. 2015;10(35):5

114. Starfield B, Mangin D. An international perspective on the basis for payment for performance. Qual Prim Care. 2010;18:399-404.

115. Chutka DS, Takahashi PY, Hoel RQW. Inappropriate medications for elderly patients. Mayo Clin Proc. 2004;79(1):122-39.

116. Morton A. Inappropriately defining "inappropriate medication for the elderly". J Am Geriatr Soc. 2004;52(9):1580.

117. Steinman MA, et al. Agreement between drugs-to-avoid criteria and expert assessments of problematic prescribing. Arch Intern Med. 2009;169(14):132632.

118. de Souto Barreto P, Ferrandez AM, Saliba-Serre B. Are older adults who volunteer to participate in an exercise study fitter and healthier than nonvolunteers? The participation bias of the study population. J Phys Act Health. 2013;10(3):359-67.

119. Golomb BA, et al. The older the better: are elderly study participants more non-representative? A cross-sectional analysis of clinical trial and observational study samples. BMJ Open. 2012;2(6): e000833.

120. Kaiser C, et al. Selection bias of elderly patients with chronic angina referred for catheterization. Int $\mathrm{J}$ Cardiol. 2006;110(1):80-5.

121. Sugisawa H, Kishino H, Sugihara Y, Okabayashi H, Shibata H Comparison of characteristics between respondents and nonrespondents in a national survey of Japanese elderly using six year follow-up study. Nihon Koshu Eisei Zasshi. 1999;46(7):551-62 (in Japanese).

122. Sugisawa H, Kishino H, Sugihara Y, Shibata H. Characteristics of dropouts and participants in a twelve-year longitudinal research of Japanese elderly. Nihon Koshu Eisei Zasshi. 2000;47(4):337-49 (in Japanese).

123. Boyd CM, et al. Clinical practice guidelines and quality of care for older patients with multiple comorbid diseases: implications for pay for performance. JAMA. 2005;294(6):716-24.

124. Tinetti ME, Bogardus ST Jr, Agostini JV. Potential pitfalls of disease-specific guidelines for patients with multiple conditions. N Engl J Med. 2004;351(27):2870-4.

125. Starfield B, Gérvas J, Mangin D. Clinical care and health disparities. Annu Rev Public Health. 2012;33:89-106.

126. Angell M. The truth about drug companies: how they deceive us and what to do about it. Random House; 2005.

127. Golomb BA. Conflict of Interest in Medicine. 5 Oct 2008. Available from: http://thesciencenetwork.org/programs/beyondbelief-candles-in-the-dark/beatrice-golomb. Accessed 2 May 2018.

128. Gotzsche P. Deadly medicines and organised crime. London: Radcliffe Medical Press; 2013.

129. Healy D. Let them eat Prozac: the unhealthy relationship between the pharmaceutical industry and depression. New York: New York University Press; 2006.

130. Healy D. Pharmageddon. California: University of California Press; 2012.

131. Healy D, Cattel D. Interface between authorship, industry and science in the domain of therapeutics. $\mathrm{Br} \mathrm{J}$ Psychiatry. 2003;183(1):22-7.

132. Doshi P, et al. Restoring invisible and abandoned trials: a call for people to publish the findings. BMJ. 2013;346:f2865.

133. Chan A, et al. Empirical evidence for selective reporting of outcomes in randomized trials: comparison of protocols to published articles. J Am Med Assoc. 2004;291(20):2457-65.

134. Bero L, et al. Factors associated with findings of published trials of drug-drug comparisons: why some statins appear more efficacious than others. PLoS Med. 2007;4(6):e184.

135. Ciani $\mathrm{O}$, et al. Comparison of treatment effect sizes associated with surrogate and final patient relevant outcomes in randomised controlled trials: meta-epidemiological study. BMJ 2013;346:f457.

136. Choudhry NK, Stelfox HT, Detsky AS. Relationships between authors of clinical practice guidelines and the pharmaceutical industry. J Am Med Assoc. 2002;287(5):612-7.

137. Gordon J. The under-representation of elderly patients in a problem-based medical school curriculum. Med Teach. 2007;29(8):844

138. Rochon PA, Gurwitz JH. Optimising drug treatment for elderly people: the prescribing cascade. BMJ. 1997;315(7115):1096-9.

139. Gill SS, et al. A prescribing cascade involving cholinesterase inhibitors and anticholinergic drugs. Arch Intern Med. 2005;165(7):808-13.

140. Anderson K, et al. Prescriber barriers and enablers to minimising potentially inappropriate medications in adults: a systematic review and thematic synthesis. BMJ Open. 2014;4(12):e006544.

141. Reeve E, et al. Patient barriers to and enablers of deprescribing: a systematic review. Drugs Aging. 2013;30(10):793-807. 
142. Checkland K, et al. Biomedicine, holism and general medical practice: responses to the 2004 General Practitioner contract. Sociol Health Illn. 2008;30(5):788-803.

143. Marengoni A, et al. Prevalence of chronic diseases and multimorbidity among the elderly population in Sweden. Am J Public Health. 2008;98(7):1198-200.

144. Barnett K, et al. Epidemiology of multimorbidity and implications for health care, research, and medical education: a crosssectional study. Lancet. 2012;380(9836):37-43.
145. Mangin D, Sweeney K, Heath I. Preventive health care in elderly people needs rethinking. BMJ. 2007;335(7614):285-7.

146. Multimorbidity: clinical assessment and management, National Institute For Health and Care Excellence guideline [NG56]. 2016: United Kingdom nice.org.uk/guidance/ng56. 